\title{
Composição faunística de vespas (Hymenoptera: Vespoidea) na Floresta Estacional do Parque Nacional da Serra da Bodoquena, Brasil
}

\author{
Tiago Henrique Auko \& Rogério Silvestre ${ }^{2,3}$ \\ ${ }^{1}$ Laboratório de Ecologia de Hymenoptera - HECOLAB, Programa de Pós-graduação em Entomologia e \\ Conservação da Biodiversidade - PPGECB, Universidade Federal da Grande Dourados - UFGD, \\ Rod. Dourados-Itahum, Km 12, Cidade Universitária, CEP 79804-970, Dourados, MS, Brasil \\ http://www.ufgd.edu.br/. \\ ${ }^{2}$ Laboratório de Ecologia de Hymenoptera - HECOLAB, Programa de Pós-graduação em Entomologia \\ e Conservação da Biodiversidade - PPGECB, Faculdade de Ciências Biológicas e Ambientais, \\ Universidade Federal da Grande Dourados - UFGD, Rod. Dourados-Itahum, Km 12, \\ Cidade Universitária, CEP 79804-970, Dourados, MS, Brasil \\ ${ }^{3}$ Autor para correspondência: Rogério Silvestre, e-mail: rogeriosilvestre@ufgd.edu.br
}

\begin{abstract}
AUKO, T.H. \& SILVESTRE, R. Faunal composition of wasps (Hymenoptera: Vespoidea) in a seasonal forest from Serra da Bodoquena National Park, Brazil. Biota Neotrop. 13(1): http://www.biotaneotropica.org.br/ v13n1/en/abstract?inventory+bn03013012013
\end{abstract}

Abstract: This work aims at performing the inventory of predator wasps' fauna (Hymenoptera, Vespoidea) from Serra da Bodoquena National Park, Brazil, municipalities of Bodoquena, Bonito, Jardim and Porto Murtinho; with a discontinuous period of 42 days of collecting, between February/2007 to December/2008. Seven expeditions were undertaken in the field in areas of deciduous and semi deciduous seasonal forest. The techniques employed in faunal inventory were: active collection with entomological net, Malaise traps, Möericke traps and hand collecting. Three-hundred sixty one specimens in Vespoidea group were captured, represented by 107 species in 59 genera, distributed in the following families: Vespidae, Pompilidae, Mutillidae, Tiphiidae and Scoliidae. Vespidae were the most frequently sampled group. Pompilidae presented a wider range of records in relation to collection methods; however most genera are sampled by an exclusive technique. Scoliidae was only represented by tree individuals. Polybia (gr. occidentalis) and Agelaia multipicta (Vespidae) were the most abundant between all the Aculeata wasps sampled. Sixty one species was sampled with only one individual (singletons). This data input new records of distribution of wasp's species to Central Western Brazil with biogeographical interest.

Keywords: aculeate wasps, community structure, diversity, dry forest, quick inventory.

AUKO, T.H. \& SILVESTRE, R. Composição faunística de vespas (Hymenoptera: Vespoidea) na Floresta Estacional do Parque Nacional da Serra da Bodoquena, Brasil. Biota Neotrop. 13(1): http://www. biotaneotropica.org.br/v13n1/pt/abstract?inventory+bn03013012013

Resumo: Neste trabalho realizamos um inventário da fauna de vespas predadoras (Hymenoptera, Vespoidea) do Parque Nacional da Serra da Bodoquena (PNSB), municípios de Bodoquena, Bonito, Jardim e Porto Murtinho, em 42 dias descontínuos de amostragens entre fevereiro de 2007 a dezembro de 2008. Sete expedições de campo foram realizadas em áreas de Floresta Estacional Decidual e Floresta Estacional Semidecidual. As técnicas empregadas foram: coletas ativas com rede entomológica, armadilha de Malaise, bandejas amarelas (armadilha de Möericke) e coleta manual. Trezentos e sessenta e um indivíduos de Vespoidea foram capturados, representados por 107 espécies, em 59 gêneros, distribuídos nas seguintes famílias: Vespidae, Pompilidae, Mutillidae, Tiphiidae e Scoliidae. Vespidae foi o grupo mais freqüente. Pompilidae foi bem representado por um amplo conjunto de métodos de coleta, entretanto a maioria dos gêneros foi amostrada por uma técnica exclusiva. Scoliidae foi representado apenas por três indivíduos. As espécies Polybia (gr. occidentalis) e Agelaia multipicta (Vespidae) foram as mais abundantes dentre todas as vespas aculeadas amostradas. Sessenta e uma espécies capturadas estão representadas por apenas um único indivíduo (singletons). Estes dados acrescentam novos registros de distribuição de espécies de vespas para a região Centro-Oeste do Brasil de interesse biogeográfico.

Palavras-chave: vespas aculeadas, estrutura da comunidade, diversidade, mata seca, inventário rápido. 


\section{Introdução}

A estrutura da comunidade de vespas ainda é pouco compreendida para a região Neotropical e inventários rápidos da diversidade desse grupo contribuem para os diagnósticos faunísticos e inferem informações biogeográficas relevantes em ambientes nunca antes amostrados e que são hotspots de diversidade. Muitos táxons carecem ainda de revisão, não existindo uma classificação de consenso entre os pesquisadores. Atributos como o comportamento, que vai do social ao solitário, a grande variedade de hábitats que ocupam e a grande amplitude sazonal e altitudinal, conferem a estas vespas um "sucesso" ecológico na exploração de recursos, e as tornam importantes agentes estruturadores das comunidades (Sarmiento 1997).

Pilgrim et al. (2008) acreditam que Vespoidea seja um grupo parafilético e propõem uma nova classificação dos Hymenoptera Aculeata, reconhecendo oito superfamílias: Apoidea, Chrysidoidea, Formicoidea, Pompiloidea, Scolioidea, Tiphioidea, Thynnoidea e Vespoidea.

Vespoidea (senso Brothers \& Carpenter, 1999) é formada por 10 famílias com aproximadamente 50.000 espécies descritas no mundo. Na Região Neotropical estão registrados cerca de 2.500 gêneros e mais de 24.000 espécies descritas (Fernández 2002).

Vespidae abarca mais de 4.500 espécies descritas, em 268 gêneros e sete subfamílias. Está distribuído em todo o mundo, sendo a maior riqueza encontrada nos trópicos. Apenas três subfamílias são encontradas no Brasil, sendo elas Masarinae, Eumeninae e Polistinae (Carpenter \& Marques 2001).

Pompilidae abrange 4.200 espécies distribuídas pelo mundo (Brothers \& Finnamore 1993), na região Neotropical encontram-se aproximadamente 750 espécies (Fernández 2000). As fêmeas utilizam aranhas como presas para alimentarem suas larvas, sendo que cada larva se alimenta de uma única presa (Brothers \& Carpenter 1993).

Mutillidae possui aproximadamente 208 gêneros e 4.200 espécies descritas, estima-se que este número deva chegar próximo a 6.000 espécies (Lelej 2007, Lelej \& Brothers 2008). Na região Neotropical são encontradas duas das sete subfamílias existentes: Sphaerophtalminae e Mutillinae. Essas vespas comportam-se como parasitóides de outros himenópteros aculeados, principalmente de abelhas solitárias (Brothers 2006).

Tiphiidae são vespas cosmopolitas e predominantes em regiões tropicais. A família contém cerca de 1500 espécies distribuídas em sete subfamílias, e provavelmente todos os Tiphiidae sejam parasitóides de estádios larvais de coleópteros de solo (Scarabaeidae, Curculionidae, Carabidae, Tenebrionidae), com exceção de um gênero Australiano Diamma que se alimenta de grilos (Kimsey \& Brothers 2006).

Scoliidae também são cosmopolitas. Todas as espécies são solitárias. A família contém cerca de 300 espécies e cinco gêneros no mundo (Brothers \& Finnamore 1993). As fêmeas são ectoparasitóides de larvas de besouros (Scarabaeidae, Passalidae, Lucanidae) e suas pernas anteriores fortes permitem escavar o solo em busca das larvas destes coleópteros (Fernández 2006).

Levantamentos recentes de vespas (Vespoidea) no Brasil são poucos com inventários de grupos isolados, principalmente envolvendo vespas sociais. Silveira (2002) realizou um inventário para a região Amazônica no Pará; Somavilla et al. (2010) avaliaram a diversidade de vespas para os Pampas gaucho; Santos et al. (2009) trabalhou com a região do Cerrado na Bahia; Togni (2009) inventariou a Mata Atlântica no estado de São Paulo e Santos (2008), também na Mata Atlântica avaliou a diversidade de Vespoidea solitários em um gradiente altitudinal em São Paulo.

A Serra da Bodoquena faz parte do corredor ecológico de Biodiversidade Cerrado-Pantanal, pertencendo à zona núcleo da Reserva da Biosfera da Mata Atlântica e da Biosfera do Pantanal e está sendo altamente ameaçada pela exploração turística na região de Bonito e pelas fazendas adjacentes (Brasil 2007). A fauna desta região ainda é pouco conhecida, com exceção da comunidade de anuros (Uetanabaro et al. 2007) e formigas (Silvestre et al. 2012).

O Objetivo deste trabalho foi inventariar a diversidade de vespas do Parque Nacional da Serra da Bodoquena, região esta com grande importância geológica e biogeográfica, nunca antes amostrada para este grupo de insetos.

\section{Material e Métodos}

\section{Sítios amostrados}

O Parque Nacional da Serra da Bodoquena (Figura 1) está localizado na porção Centro - Oeste do país, neste planalto encontrase um dos últimos remanescente de Floresta Estacional Decidual (Mata Seca) e Semidecidual (Mata Atlântica) do país com qualidade preservada e com grande extensão (Pott \& Pott 2003).

Realizamos ao todo sete expedições de coleta, com duração de cinco dias cada. Os pontos de coletas foram principalmente em áreas que compunham as sub-bacias dos rios Salobra e Perdido, da bacia do rio Paraguai. Os nomes das localidades são referentes às fazendas limítrofes ao PNSB (Tabela 1).

\section{Métodos de coleta}

Para o inventário rápido da diversidade de vespas na Serra da Bodoquena foram empregados os seguintes métodos:

1. Coleta ativa (rede entomológica e pinça): Nesta metodologia incluem-se a observação em lugares específicos como barrancos de rios e ranchos abandonados, que apresentam condições físicas propícias para as construções de ninhos de vespas, além da observação da vegetação.

Tabela 1. Área amostrada, altitude, data e coordenadas geográficas das sete expedições de coletas de vespas realizadas no Parque Nacional da Serra da Bodoquena, em um período descontínuo de 42 dias de coleta de campo, entre fevereiro/2007 a dezembro/2008.

Table 1. Sampled area, altitude, date and geographical coordinates of the seven expeditions collections of wasps held in Serra da Bodoquena National Park, in a discontinuous period of 42 days of field collecting, during February 2007 to December 2008.

\begin{tabular}{clccl}
\hline Expedição & Área amostrada & Altitude & Data & Coordenadas \\
\hline I & Califórnia & $610 \mathrm{~m}$ & fev./2007 & $20^{\circ} 42^{\prime} 07^{\prime \prime} \mathrm{S} ; 56^{\circ} 52^{\prime} 47^{\prime} \mathrm{W}$ \\
II & Marambaia & $570 \mathrm{~m}$ & maio/2007 & $20^{\circ} 56^{\prime} 56^{\prime \prime} \mathrm{S} ; 56^{\circ} 47^{\prime} 75^{\prime} \mathrm{W}$ \\
III & Santa Laura I & $225 \mathrm{~m}$ & nov. $/ 2007$ & $20^{\circ} 46^{\prime} 56^{\prime \prime} \mathrm{S} ; 56^{\circ} 44^{\prime} 31^{\prime} \mathrm{W}$ \\
IV & Santa Maria & $390 \mathrm{~m}$ & fev./2008 & $21^{\circ} 32^{\prime} 46^{\prime \prime} \mathrm{S} ; 56^{\circ} 55^{\prime} 29^{\prime} \mathrm{W}$ \\
V & Santa Laura II & $225 \mathrm{~m}$ & abr./2008 & $20^{\circ} 46^{\prime} 56^{\prime \prime} \mathrm{S} ; 56^{\circ} 44^{\prime} 31^{\prime \prime} \mathrm{W}$ \\
VI & Pitangueiras & $490 \mathrm{~m}$ & abr./2008 & $20^{\circ} 52^{\prime} 13^{\prime \prime} \mathrm{S} ; 56^{\circ} 35^{\prime} 20^{\prime} \mathrm{W}$ \\
VII & Campo Verde & $470 \mathrm{~m}$ & dez./2008 & $21^{\circ} 22^{\prime} 49^{\prime \prime} \mathrm{S} ; 56^{\circ} 45^{\prime} 46^{\prime} \mathrm{W}$ \\
\hline
\end{tabular}




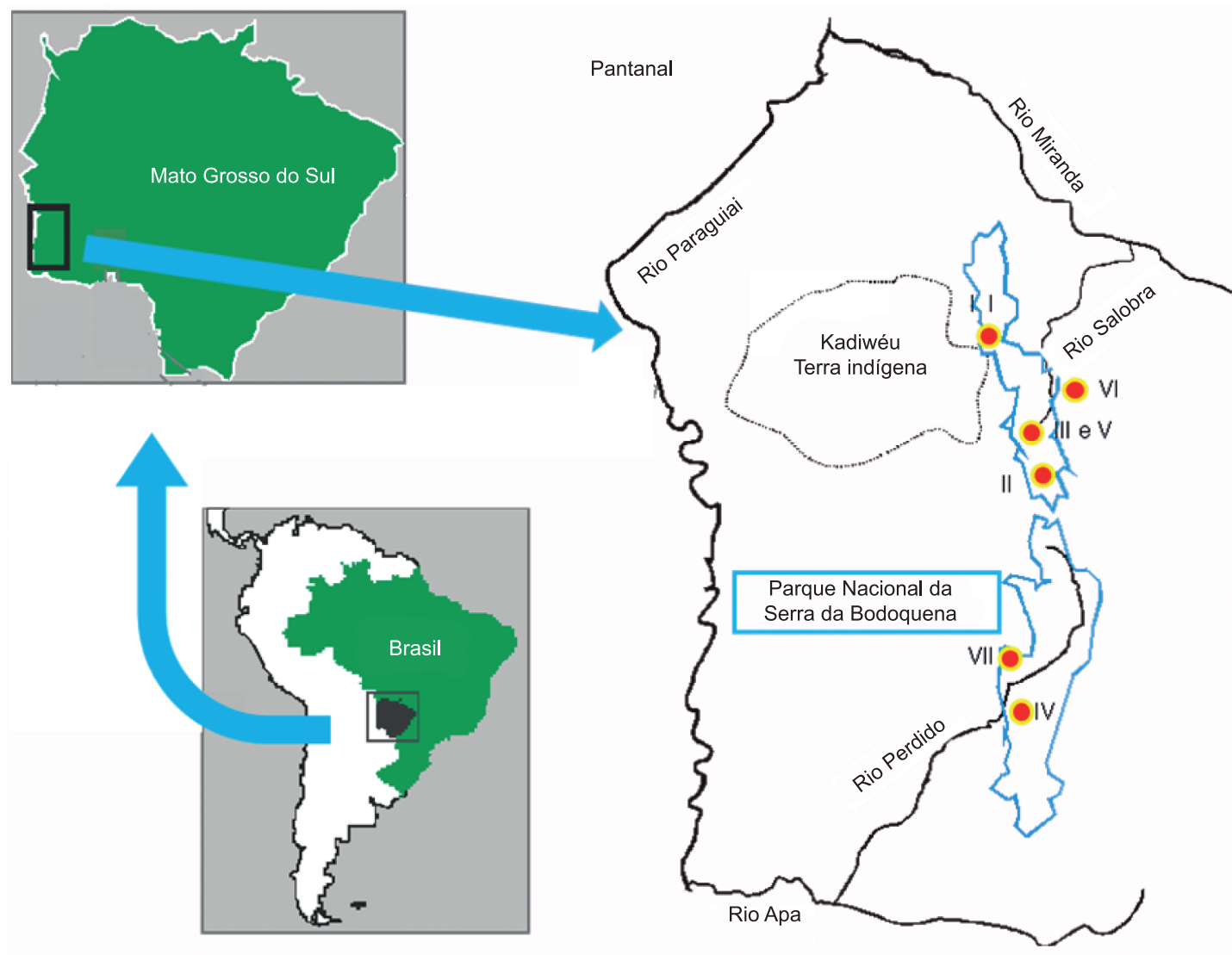

Figura 1. Localização do Parque Nacional da Serra da Bodoquena (traço azul). Os círculos indicam os pontos de coleta de vespas (Hymenoptera: Vespoidea). Figure 1. Location of Serra da Bodoquena National Park (blue line). The circles show the points of collection wasps (Hymenoptera: Vespoidea).

2. Armadilhas (Möericke): Ao todo foram dispostas 350 bandejas, sendo 50 por expedição de coleta, distando $10 \mathrm{~m}$ uma armadilha da outra.

3. Armadilha de Malaise: Ao todo no inventário foram recolhidas 14 amostras, dispostas sobre o solo por quatro dias consecutivos.

O material foi comparado com os espécimes depositados na coleção referência do Museu de Zoologia da USP de São Paulo e alguns exemplares foram determinados por especialistas de seus respectivos grupos. Os exemplares estão depositados na Coleção de Hymenoptera do Museu de Biodiversidade da UFGD (MuBio), em Dourados - MS.

\section{Análises}

A estimativa de riqueza de espécies foi obtida pela curva de rarefação (Simberloff 1972) para as famílias mais abundantes, calculado a partir da presença e ausência das espécies nas amostras. Foi empregado para este teste o pacote estatístico EstimateS, versão 8.0 (Colwell 2004).

\section{Resultados}

Foram coletados 361 espécimes de Vespoidea (excluindo as formigas), pertencentes a 107 espécies em 59 gêneros (Tabela 2), um gênero dentre os Pompilini não foi identificado. Estas espécies estão classificadas em cinco famílias, sendo elas: Vespidae (37), Pompilidae (40), Mutillidae (22), Tiphiidae (5) e Scoliidae (3).

As espécies Polybia (gr. occidentalis) e Agelaia multipicta (Vespidae) foram as mais abundantes dentre todos os aculeados amostrados. Sessenta e uma espécies amostradas (58\%) estão representadas por apenas um único indivíduo (singletons).

A Figura 2 apresenta a estimativa de riqueza de espécies para as famílias mais abundantes: Vespidae, Mutillidae e Pompilidae, dentre os himenópteros vespóideos amostrados.

Vinte e quatro espécies, distribuídas em 18 gêneros, em cinco famílias foram capturadas em armadilhas de Malaise. Nas bandejas amarelas foram 22 espécies distribuídas em 16 gêneros e quatro famílias, sendo que apenas Ageniella sp. 1 e Episyron sp. (Pompilidae), Timulla spp. đ̂ e Ephuta spp. ô (Mutillidae), ocorreram nas duas armadilhas. As armadilhas amarelas e as de Malaise juntas amostraram 42 espécies, representando $40 \%$ da fauna total de vespas aculeadas do trabalho. A coleta de rede entomológica e manual com pinça, ao longo de trilhas na mata e margens de rios e córregos, amostrou um total de 63 espécies de Vespoidea, representando $60 \%$ do total (Tabela 3).

Neste trabalho Sierolomorphidae, Rhopalosomatidae, Sapygidae e Bradynobaenidae não foram amostrados. Sendo que o primeiro grupo está registrado somente na América Central e os dois últimos para o Chile e a Argentina, com poucas espécies relatadas. Para Rhopalosomatidae são registrados apenas três gêneros no Brasil (Sarmiento 2006).

\section{Discussão}

Os resultados da coleta de Vespoidea obtidos neste estudo mostraram que a região na qual se insere o P.N.S.B. é consideravelmente diversa em relação a outros levantamentos na região Neotropical. Santos (2008) utilizando metodologia semelhante obteve uma 
Vespas da Serra da Bodoquena

Tabela 2. Lista de espécies de Vespoidea amostrados no PNSB, Brasil, em sete expedições de coleta.

Table 2. The species list for Vespoidea sampled in PNSB, Brazil, in seven collecting expeditions.

\begin{tabular}{|c|c|c|c|c|c|c|c|}
\hline \multirow{2}{*}{ Espécies } & \multicolumn{7}{|c|}{ Expedições } \\
\hline & $\mathbf{I}$ & II & III & IV & $\mathbf{V}$ & VI & VII \\
\hline \multicolumn{8}{|l|}{ Vespidae } \\
\hline \multicolumn{8}{|l|}{ Eumeninae } \\
\hline Ancistroceroides conjunctus (Fox) & - & - & 2 & - & - & - & 1 \\
\hline Hypancistrocerus sp. & - & - & - & - & - & - & 1 \\
\hline Minixi suffusum (Fox) & - & - & - & - & - & - & 1 \\
\hline Monobia angulosa Saussure & - & - & - & - & - & - & 1 \\
\hline Montezumia azurescens (Spinola) & - & - & - & - & - & 1 & - \\
\hline Montezumia ferruginea brasiliensis Saussure & - & - & - & - & - & - & 1 \\
\hline Montezumia infernalis (Spinola) & - & - & - & 1 & - & 1 & - \\
\hline Montezumia petiolata Saussure & - & - & - & - & 2 & - & - \\
\hline Omicron spegazzinii (Brèthes, 1905) & - & - & - & - & - & - & 1 \\
\hline Omicron tuberculatum (Fox, 1899) & - & - & - & - & - & - & 3 \\
\hline Pachodynerus guadulpensis (Saussure) & - & 1 & 1 & - & 1 & - & 3 \\
\hline Pachodynerus brevithorax (Saussure) & - & - & 2 & - & - & - & - \\
\hline Pachodynerus grandis Willink \& Roig-Alsina & - & - & - & - & - & - & 1 \\
\hline Pachodynerus nasidens (Latreille) & - & - & - & - & 2 & 1 & 2 \\
\hline Pachymenes ghilianii (Spinola, 1851) & - & - & - & - & - & 1 & - \\
\hline Pachyminixi sp. 1 & - & - & - & - & - & - & 1 \\
\hline Parancistrocerus sp. 1 & - & - & - & - & - & - & 1 \\
\hline Parancistrocerus sp. 2 & - & - & - & - & - & 1 & - \\
\hline Zeta argillaceum (Linnaeus) & - & - & 1 & - & 1 & 2 & - \\
\hline Zethus cylindricus Fox & - & - & - & 3 & - & - & 2 \\
\hline \multicolumn{8}{|l|}{ Polistinae } \\
\hline \multicolumn{8}{|l|}{ Epiponini } \\
\hline Agelaia multipicta Haliday, 1836 & - & 1 & 3 & 8 & - & 3 & 4 \\
\hline Apoica flavissima van der Vecht & - & - & - & - & - & 1 & - \\
\hline Brachygastra lecheguana (Latreille) & - & - & - & - & - & - & 1 \\
\hline Brachygastra moulae Richards, 1978 & - & - & 2 & - & - & - & 9 \\
\hline Metapolybia sp. & - & - & - & - & - & 1 & - \\
\hline Polybia chrysothorax (Lichtenstein) & - & - & 1 & 2 & 1 & 8 & - \\
\hline Polybia ignobilis (Haliday) & 2 & - & 2 & - & 2 & 4 & 5 \\
\hline Polybia (gr. occidentalis) (Oliver) & - & 1 & - & 8 & - & 2 & 11 \\
\hline Protonectarina silveirae (Saussure) & - & - & 1 & - & - & - & 3 \\
\hline Protopolybia exigua exigua (Saussure) & - & - & - & - & - & - & 1 \\
\hline \multicolumn{8}{|l|}{ Mischocyttarini } \\
\hline Mischocyttarus sp. 1 & - & - & - & - & - & 1 & - \\
\hline Mischocyttarus sp. 2 & - & - & 3 & - & 3 & 1 & - \\
\hline Mischocyttarus sp. 3 & - & - & - & - & - & 1 & 1 \\
\hline Mischocyttarus sp. 4 & - & - & - & - & 1 & - & 1 \\
\hline \multicolumn{8}{|l|}{ Polistini } \\
\hline Polistes canadensis (Linnaeus) & 2 & 2 & - & - & - & 6 & 2 \\
\hline Polistes ferreri Saussure, 1853 & - & - & - & - & - & - & 4 \\
\hline Polistes simillimus Zikan & - & 2 & - & 3 & - & - & 4 \\
\hline Polistes versicolor (Olivier) & - & 5 & - & - & - & - & 1 \\
\hline \multicolumn{8}{|l|}{ Mutillidae } \\
\hline \multicolumn{8}{|l|}{ Sphaerophtalminae } \\
\hline \multicolumn{8}{|l|}{ Sphaerophtalmini } \\
\hline Atillum sp. & - & - & - & - & - & 1 & - \\
\hline Hoplocrates monacha (Gerstaecker) & - & 1 & - & - & - & - & - \\
\hline Hoplocrates sp. 2 & 1 & - & - & - & - & - & - \\
\hline Mickelia sp. 2 & 1 & - & - & - & - & - & - \\
\hline Ptilomutilla pennata André, 1905 & - & - & - & 1 & - & - & - \\
\hline
\end{tabular}


Tabela 2. Continuação...

\begin{tabular}{|c|c|c|c|c|c|c|c|}
\hline \multirow{2}{*}{ Espécies } & \multicolumn{7}{|c|}{ Expedições } \\
\hline & $\mathbf{I}$ & II & III & IV & $\mathbf{V}$ & VI & VII \\
\hline Sphinctopsis turnalia (Cresson) & - & - & 1 & - & - & - & - \\
\hline Sphinctopsis sp. 2 & 1 & - & - & 1 & - & - & - \\
\hline Sphinctopsis sp. 3 & - & - & - & 1 & - & - & - \\
\hline Suareztilla sp. & 1 & - & - & - & - & - & - \\
\hline Traumatomutilla manca (Cresson) & 1 & - & - & - & - & 1 & - \\
\hline Traumatomutilla graphica (Gerstaecker) & 1 & - & - & 1 & - & - & - \\
\hline Traumatomutilla sp. 1 & - & - & - & 1 & - & 1 & - \\
\hline Traumatomutilla sp. 2 & 1 & - & - & - & - & - & - \\
\hline Traumatomutilla sp. 3 & 1 & - & - & - & - & - & - \\
\hline Traumatomutilla sp. 4 & - & - & - & - & - & 1 & - \\
\hline Traumatomutilla sp. 6 & - & - & 1 & - & - & - & - \\
\hline Traumatomutilla sp. 15 & - & - & - & - & - & - & 1 \\
\hline \multicolumn{8}{|l|}{ Mutillinae } \\
\hline \multicolumn{8}{|l|}{ Ephutini } \\
\hline Ephuta spp. $\lesssim$ & 2 & 1 & 2 & 2 & 2 & - & 6 \\
\hline \multicolumn{8}{|l|}{ Mutillini } \\
\hline Timulla sp. 1 & - & - & - & 1 & - & 1 & - \\
\hline Timulla sp. 2 & - & - & - & 1 & - & - & 1 \\
\hline Timulla sp. 3 & - & - & - & - & - & - & 1 \\
\hline Timulla spp. $\widehat{o}$ & - & - & 1 & 4 & - & - & 1 \\
\hline \multicolumn{8}{|l|}{ Pompilidae } \\
\hline \multicolumn{8}{|l|}{ Pepsinae } \\
\hline \multicolumn{8}{|l|}{ Ageniellini } \\
\hline Ageniella sp. 1 & - & 2 & 3 & - & - & - & - \\
\hline Ageniella sp. 2 & - & - & - & - & - & 1 & - \\
\hline Auplopus sp. & - & - & - & 8 & - & 1 & - \\
\hline Priocnemella sp. & - & - & - & - & - & 1 & - \\
\hline \multicolumn{8}{|l|}{ Pepsini } \\
\hline Aimatocare sp. 1 & - & - & 1 & - & - & - & - \\
\hline Aimatocare sp. 2 & - & - & - & 1 & - & - & - \\
\hline Caliadurgus sp. & 1 & 1 & 2 & - & 3 & 1 & - \\
\hline Entypus sp. 1 & - & - & - & - & - & 1 & - \\
\hline Entypus sp. 2 & - & - & - & - & - & - & 1 \\
\hline Entypus sp. 3 & - & - & - & - & - & - & 1 \\
\hline Pepsis crassicornis Mócsary, 1885 & - & - & - & - & - & 1 & - \\
\hline \multicolumn{8}{|l|}{ Ctenocerinae } \\
\hline Epipompilus aztecus (Cresson) & - & - & 1 & - & - & - & - \\
\hline Epipompilus sp. & - & - & 1 & - & - & - & - \\
\hline \multicolumn{8}{|l|}{ Ceropalinae } \\
\hline Ceropales sp. & - & - & - & - & - & - & 1 \\
\hline \multicolumn{8}{|l|}{ Pompilinae } \\
\hline \multicolumn{8}{|l|}{ Aporini } \\
\hline Aporus sp. & - & - & - & - & - & 1 & - \\
\hline Euplaniceps sp. 1 & - & - & - & 2 & - & - & 1 \\
\hline Euplaniceps sp. 2 & - & - & - & 1 & - & - & - \\
\hline Notoplaniceps sp. & - & - & - & - & - & - & 1 \\
\hline \multicolumn{8}{|l|}{ Pompilini } \\
\hline Agenioideus sp. & 1 & - & - & 1 & 2 & - & - \\
\hline Anoplius taschenbergui (Brèthes) & - & - & - & 1 & - & - & - \\
\hline Anoplius sp. 2 & - & - & - & - & - & - & 4 \\
\hline Anoplius sp. 3 & - & - & - & - & - & - & 1 \\
\hline Anoplius sp. 4 & - & - & - & - & - & - & 1 \\
\hline Aplochares sp. & - & - & - & - & - & 1 & - \\
\hline
\end{tabular}


Tabela 2. Continuação...

\begin{tabular}{|c|c|c|c|c|c|c|c|}
\hline \multirow{2}{*}{ Espécies } & \multicolumn{7}{|c|}{ Expedições } \\
\hline & $\mathbf{I}$ & II & III & IV & $\mathbf{V}$ & VI & VII \\
\hline Dicranoplius sp. & - & - & - & - & - & - & 1 \\
\hline Episyron sp. & - & - & - & 1 & - & - & - \\
\hline Paracyphononyx sp. & - & - & - & - & - & 5 & - \\
\hline Poecilopompilus sp. 1 & 1 & - & - & - & - & - & - \\
\hline Poecilopompilus sp. 2 & - & - & - & 3 & - & - & - \\
\hline Poecilopompilus sp. 3 & - & - & 1 & - & - & - & - \\
\hline Poecilopompilus sp. 4 & - & - & 1 & - & - & - & - \\
\hline Poecilopompilus sp. 5 & - & - & - & - & - & 1 & - \\
\hline Priochilus sp. 1 & 4 & - & 1 & - & - & 1 & - \\
\hline Priochilus sp. 2 & - & - & 1 & - & - & - & - \\
\hline Priochilus gracillimus Smith, 1855 & - & - & 2 & - & - & 1 & - \\
\hline Notocyphus sp. 1 & - & - & 1 & - & - & - & - \\
\hline Notocyphus sp. 2 & - & - & 1 & - & - & - & - \\
\hline Notocyphus sp. 3 & - & - & - & - & - & - & 1 \\
\hline Tachypompilus sp. & - & - & - & - & 5 & 1 & - \\
\hline Pompilini sp. & - & - & - & 1 & - & - & - \\
\hline \multicolumn{8}{|l|}{ Scoliidae } \\
\hline \multicolumn{8}{|l|}{ Campsomerinae } \\
\hline Campsomeris terrestres (Saussure) & - & - & - & - & - & - & 1 \\
\hline Campsomeris sp. 1 & - & - & - & - & - & 1 & - \\
\hline Campsomeris sp. 2 & - & - & - & - & - & - & 1 \\
\hline \multicolumn{8}{|l|}{ Tiphiidae } \\
\hline \multicolumn{8}{|l|}{ Myzininae } \\
\hline Myzinum sp. & - & - & - & - & - & - & 1 \\
\hline Pterombrus sp. & - & - & - & 4 & - & - & - \\
\hline \multicolumn{8}{|l|}{ Tiphiinae } \\
\hline Epomidiopteron aspilata (Banks) & - & - & - & - & - & - & 1 \\
\hline Tiphia sp. 1 & 9 & 1 & - & 4 & - & 2 & - \\
\hline Tiphia sp. 2 & 1 & - & - & - & - & - & - \\
\hline Total $n^{\circ}$ de espécies/localidade & 31 & 18 & 39 & 65 & 25 & 60 & 93 \\
\hline
\end{tabular}

Tabela 3. Número de indivíduos amostrados pelas diferentes técnicas de coleta do grupo Vespoidea no Parque Nacional da Serra da Bodoquena no período de janeiro de 2007 a dezembro de 2008.

Table 3. Number of individuals sampled by different collection techniques of Vespoidea group in Serra da Bodoquena National Park from January 2007 to December 2008.

\begin{tabular}{|c|c|c|c|c|c|}
\hline Famílias & Rede entomológica & Manual & Malaise & Möerick & Total \\
\hline Vespidae & 159 & - & 12 & 3 & 174 \\
\hline Pompilidae & 50 & - & 20 & 43 & 113 \\
\hline Mutillidae & - & 26 & 12 & 10 & 48 \\
\hline Tiphiidae & 6 & - & 5 & 12 & 23 \\
\hline Scoliidae & 2 & - & 1 & - & 3 \\
\hline Total & 215 & 26 & 50 & 68 & 361 \\
\hline
\end{tabular}

abundância de 817 exemplares, distribuídos em 76 espécies de Vespoidea (excluindo Polistinae) na Mata Atlântica. Morato et al. (2008) utilizando apenas armadilhas de Malaise amostraram 45 espécies de Vespoidea, distribuídas em 18 gêneros, com um total de 366 indivíduos coletados na região do Parque Nacional da Serra do Divisor no Acre. Mechi (1996) realizou inventário de Vespoidea em duas áreas de Cerrado em São Paulo e encontrou uma abundância de 2.200 indivíduos com uma riqueza de 105 espécies. Silveira (2002) fazendo um inventário de Polistinae na Amazônia com a utilização de Malaise e coleta ativa com rede entomológica amostrou 79 espécies em um período de um ano, enfatizando que $2 / 3$ da diversidade das vespas do Brasil está nessa região Amazônica e grande parte desta fauna é endêmica. No Parque Nacional Chapada dos Guimarães, no estado de Mato Grosso, Diniz \& Kitayama (1998) encontraram 30 espécies em 15 gêneros da família Vespidae, com esforço de amostragem de cinco semanas.

Silveira (2002) também observou que com a coleta ativa se obtêm um maior número de espécies, porém algumas espécies são amostradas apenas nas armadilhas de Malaise. A Curva de rarefação (Jacknife 2) para as famílias Mutillidae, Pompilidae e Vespidae, nos 


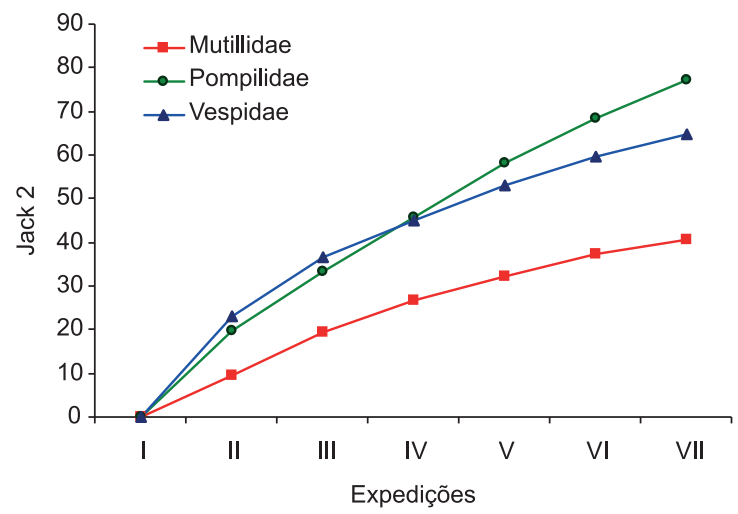

Figura 2. Curva de rarefação (Jacknife 2) para as famílias Mutillidae, Pompilidae e Vespidae, nos sete sítios amostrados no Parque Nacional da Serra da Bodoquena.

Figure 2. Rarefaction curve (Jacknife 2) for families Mutillidae, Pompilidae and Vespidae, in seven sampled sites in Serra da Bodoquena National Park.

sete sítios amostrados no Parque Nacional da Serra da Bodoquena, mostra que as coletas ainda não foram suficientes para estabilizar as curvas.

Nos diferentes registros por localidade observamos que existe uma diferença muito acentuada no número de espécies capturadas; isto mostra uma grande sazonalidade da fauna nesta região coberta por Floresta Estacional. O número de registros com rede entomológica indica que a experiência do coletor influencia nos resultados obtidos e que os métodos são complementares.

As fêmeas da família Mutillidae foram exclusivamente capturadas manualmente, com o auxílio de pinças e os machos foram capturados em armadilhas de Malaise e em bandejas amarelas. Em geral os Mutillidae são coletados em locais relativamente arenosos, como savanas e caatingas, que são áreas onde se encontram seus hospedeiros mais comuns (Brothers 2006).

O primeiro registro de Epipompilus aztecus (Pompilidae) para a América do Sul (Silvestre et al. 2010) foi obtido neste levantamento. Este inventário contempla novos registros de espécies e gêneros para a região Centro-Oeste, ampliando os registros de distribuição das espécies de vespas (Vespoidea) e gerando novas informações para estudos biogeográficos do grupo.

Nossos resultados apontam para uma alta beta diversidade de vespas para a Serra da Bodoquena, mostrando que a região tem um importante papel na distribuição desta fauna para a região CentroOeste.

\section{Agradecimentos}

Ao CNPq e à FUNDECT/MS pelo apoio financeiro. Eduardo F. dos Santos, Fernando B. Noll, Bolívar Garcete-Barrett e Marcel Hermes, pela identificação das espécies. Ao Museu de Zoologia da USP, em nome do Dr. Carlos R. F. Brandão. Ao Instituto Chico Mendes de Biodiversidade de Bonito, MS, em nome de Fernando C. Villela (IBAMA - número 10674-11/09/2007).

\section{Referências Bibliográficas}

BRASIL. Ministério do Meio Ambiente. 2007. Biodiversidade Brasileira: Áreas Prioritárias para a Conservação, Uso Sustentável e Repartição de Benefícios da Biodiversidade Brasileira. MMA, Secretaria de Biodiversidade e Florestas, Brasília. http://www.mma.gov.br (último acesso em 25/04/2012).
BROTHERS, D.J. 2006. Familia Mutillidae. In Introducción a los Hymenoptera de la región Neotropical (F. Fernández \& M.J. Sharkey, eds.). Sociedad Colombiana de Entomologia y Universidad Nacional de Colombia, Bogotá, Colombia, p.577-593.

BROTHERS, D.J. \& CARPENTER, J.M. 1993. Philogeny of Aculeata: Chrysidoidea and Vespoidea (Hymenoptera). J. Hymenopt. Res. 2(1):227-304.

BROTHERS, D.J. \& FINNAMORE, A.T. 1993. Superfamily Vespoidea. In Hymenoptera of the world: An identification guide to families (H. Goulet \& J.T. Huber, eds.). Agriculture Canada, Ottawa, Canada, p.161-278.

CARPENTER, J.M. \& MARQUES, O.M. 2001. Contribuição ao estudo dos vespídeos do Brasil. Universidade Federal da Bahia, Bahia, CD ROM, 2:1-147. Série: Publicações Digitais.

COLWELL, R.K. 2004. Estimates: Statistical estimation of species richness and shared species from samples. version 8.0. User's Guide and application.

DINIZ, I.R. \& KITAYAMA, K. 1998. Seasonality of vespid species (Hymenoptera, Vespidae) in a central Brazilian Cerrado. Rev. Biol. Trop. 46:109-114.

FERNÁNDEZ, F. 2000. Avispas Cazadoras de Arañas (Hymenoptera, Pompilidae) de la Región Neotropical. Biota Colombiana 1(1):3-24.

FERNÁNDEZ, F. 2002. Filogenia y sistemática de los himenópteros con aguijón en la Región Neotropical (Hymenoptera, Vespomorpha). In Proyecto de Red Iberoamericana de Biogeografía y Entomología Sistemática (C.S. Costa, S.A. Vanin, J.M. Lobo \& A. Melic, eds.). Monografías Tercer Milenio, Zaragoza, v.2, p.101-138.

FERNÁNDEZ, F. 2006. Familia Scoliidae. In Introducción a los Hymenoptera de la Región Neotropical (F. Fernández \& M.J. Sharkey, eds.). Sociedad Colombiana de Entomologia y Universidad Nacional de Colombia, Bogotá, Colombia, p.557-558.

KIMSEY, L.S. \& BROTHERS, D.J. 2006. Familia Tiphiidae. In Introducción a los Hymenoptera de la Región Neotropical (F. Fernández \& M.J. Sharkey, eds.). Sociedad Colombiana de Entomologia y Universidad Nacional de Colombia, Bogotá, Colombia, p.597-608.

LELEJ, A.S. 2007. Biogeography of mutillid wasps (Hymenoptera, Mutillidae). In Studies on Hymenopterous Insects (A.P. Rasnitsyn \& V.E. Gokhman, eds). Collection of Scientific Papers. KMK Scientific Press Ltd., Moscow, p.82-111.

LELEJ, A.S. \& BROTHERS, D.J. 2008. The genus-group names of Mutillidae (Hymenoptera) and their type species, with a new genus, new name, new synonymies, new combinations and lectotypifications. Zootaxa 1889:1-79.

MECHI, M.R. 1996. Levantamento da fauna de vespas na vegetação de duas áreas de cerrado. Tese de doutorado, Universidade Federal de São Carlos, São Paulo, 237p.

MORATO, E.F., AMARANTE, S.T. \& SILVEIRA, O.T. 2008. Avaliação ecológica rápida da fauna de vespas (Hymenoptera: Aculeata) do Parque Nacional da Serra do Divisor, Acre, Brasil. Acta Amazon. 38(4):789-798. http://dx.doi.org/10.1590/S0044-59672008000400025

PILGRIM, E.M., VON DOHLEN, C.D. \& PITTS, J.P. 2008. Molecular phylogenetics of Vespoidea indicate paraphyly of the superfamily and novel relationships of its component families and subfamilies Zoolog. Scrip. 37:539-560.

POTT, A. \& POTT V.J. 2003. Espécies de fragmentos florestais de Mato Grosso do Sul. In Fragmentação Florestal e Alternativas para o de desenvolvimento rural na região Centro-Oeste (R.B. Costa, ed.). UCDB, Campo Grande, p.26-52.

SANTOS, E.F. 2008. Estrutura de assembléias de Vespoidea solitários (Insecta: Hymenoptera) ao longo de um gradiente altitudinal no Parque Estadual da Serra do Mar, São Paulo, Brasil. Dissertação de Mestrado, Universidade de São Paulo, São Paulo, 75p. 
SANTOS, G.M.M.; CRUZ, J.D.; OTTO, M.M. \& GOBBI, N. 2009. Diversidade de Vespas Sociais (Hymenoptera: Vespidae) em Áreas de Cerrado na Bahia. Neotrop. Entomol. 38(3):317-320. http://dx.doi. org/10.1590/S1519-566X2009000300003

SARMIENTO, C.E. 1997. Véspidos de Colombia (Hymenoptera: Vespidae). Tesis M. Sc., Universidad Nacional de Colombia, Intituto de Ciencias Naturales, Bogotá, Colombia.

SARMIENTO, C.E. 2006. Familia Rhopalosomatidae. In Introducción a los Hymenoptera de la Región Neotropical (F. Fernández \& M.J. Sharkey, eds.). Sociedad Colombiana de Entomologia y Universidad Nacional de Colombia, Bogotá, Colombia, p.517-519.

SILVEIRA, O.T. 2002. Surveying Neotropical social wasps. An evaluation of methods in the "Ferreira Penna" Research Station (ECFPn), in Caxiuanã, PA, Brazil (Hymenoptera, Vespidae, Polistinae). Pap. Avulsos Zool. 42(12): 299-323.

SILVESTRE, R., AUKO, T.H. \& CARBONARI, V. 2010. Insecta, Hymenoptera, Vespoidea, Pompilidae, Epipompilus aztecus (Cresson, 1869). Check list 6(3):483-484.
SILVESTRE, R., DEMÉTRIO, M.F. \& DELABIE, J.C.H. 2012. Community Structure of Leaf-Litter Ants in a Neotropical Dry Forest: A biogeographic approach to explain beta diversity. Psyche 2012: Article ID 306925, 15p. http://dx.doi.org/10.1155/2012/306925

SIMBERLOFF, D. 1972. Properties of the rarefaction diversity measurement. Am. Nat. 106:414-418. http://dx.doi.org/10.1086/282781

SOMAVILLA, A., KÖHLER, A. \& HERMES, M.G. 2010. Contribuição aos estudos dos Vespidae ocorrentes no estado do Rio Grande do Sul (Insecta, Hymenoptera). Rev. Bras. Bioci. 8(3):257-263.

TOGNI, O.C. 2009. Diversidade de Vespas Sociais (Hymenoptera, Vespidae) na Mata Atlântica do Litoral Norte do Estado de São Paulo. Dissertação de Mestrado, Universidade Estadual Paulista Júlio de Mesquita Filho, Rio Claro, São Paulo.

UETANABARO, M., SOUZA, F.L., LANDGREF FILHO, P., BEDA, A.F. \& BRANDÃO, R.A. 2007. Anfíbios e répteis do Parque Nacional da Serra da Bodoquena, Mato Grosso do Sul, Brasil. Biota Neotrop. 7(3):279-289. http://dx.doi.org/10.1590/S1676-06032007000300030

Recebido em 18/08/2011

Versão reformulada recebida em 24/05/2012

Publicado em 08/03/2013 